\title{
ULF wave effects on high frequency signal propagation through the ionosphere
}

\author{
C. L. Waters and S. P. Cox \\ School of Mathematical and Physical Sciences, The University of Newcastle, Callaghan, 2308, New South Wales, Australia
}

Received: 22 September 2008 - Revised: 31 May 2009 - Accepted: 6 July 2009 - Published: 14 July 2009

\begin{abstract}
Variations in the total electron content (TEC) of the ionosphere alter the propagation characteristics of EM radiation for frequencies above a few megahertz (MHz). Spatial and temporal variations of the ionosphere TEC influence highly sensitive, ground based spatial measurements such as those used in radio astronomy and Global Positioning System (GPS) applications. In this paper we estimate the magnitudes of the changes in TEC and the time delays of high frequency signals introduced by variations in the ionosphere electron density caused by the natural spectrum of ultra-low frequency (ULF) wave activity that originates in near-Earth space. The time delays and associated phase shifts depend on the frequency, spatial structure and amplitude of the ULF waves.
\end{abstract}

Keywords. Ionosphere (Ionospheric disturbances; Wave propagation) - Magnetospheric physics (MHD waves and instabilities)

\section{Introduction}

The terrestrial ionosphere represents an inner boundary of the space environment where the transition from the neutral atmosphere to the ionised gas (plasma state) of space occurs. The ionosphere is maintained primarily through absorption of ultra-violet (UV) and X-ray radiation of solar origin (Kelley, 1989). In broad terms, at mid-latitudes the UV and X-ray energy flux increases while neutral particle densities decrease with increasing altitude. The resulting paraboliclike variation of the free electron number density, $\left(N_{e}\right)$ with altitude forms the ionosphere layers with maximum values for $N_{e}$ around $300 \mathrm{~km}$ (F2 layer). At high latitudes in the auroral zones, ionisation by particle precipitation augments

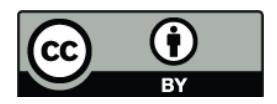

Correspondence to: C. L. Waters (colin.waters@newcastle.edu.au) the solar radiation components. Typical mid-latitude, peak daytime electron number densities are around $5 \times 10^{11} \mathrm{~m}^{-3}$ (Schunk and Nagy, 1980). All high frequency (HF), electromagnetic (EM) signals above a few $\mathrm{MHz}$ (maximum plasma frequency) from any source in space must traverse the ionosphere to reach ground based detectors.

Temporal and spatial variations in the ionosphere electron density alter the HF signal travel time. It is well known that the electron density in the ionosphere varies on seasonal and diurnal time scales. In addition to these variations, gravity waves, traveling ionosphere disturbances (TIDs), magnetic storm-time particle injections and ultra-low frequency (ULF; $1-100 \mathrm{mHz}$ ) oscillations incident from the magnetosphere perturb the ionosphere. A number of results reported during the 1970s related geomagnetic variations in the ULF band with ionosphere total electron content (TEC) fluctuations. Using the carrier phase of transmissions from the geostationary ATS 6 satellite, Davies and Hartman (1976) reported two cases where the percentage change in TEC $(\triangle$ TEC $/$ TEC $\times$ 100) was $0.03 \%$ and $0.006 \%$ with associated variations in the ULF Pc4 range (30-50 s period). A more comprehensive analysis by Okuzawa and Davies (1981) showed that variations in TEC over the 10-50 s band had a daytime bias, ranged from $0.005 \%$ up to $0.1 \%$ and similar period variations were seen in ground magnetometer records (see their Table 1).

ULF wave induced perturbations in ionosphere TEC are generally regarded to be insignificant for many applications involving high frequency EM signal propagation through the ionosphere. These include over-the-horizon radar systems, applications involving GPS signals and radio astronomy interferometry. However, as these techniques improve in resolution and signal to noise ratio the ever-present ULF oscillations become detectable and limit further improvements. For example, ULF geomagnetic variations are a source of noise in magnetic anomaly detection used in airborne geomagnetic surveys. Recent attempts to detect this ULF "noise"

Published by Copernicus Publications on behalf of the European Geosciences Union. 
using GPS measurements have shown changes in TEC units $\left(1 \mathrm{TECU}=1 \times 10^{16} e^{-} \mathrm{m}^{2}\right)$ up to a factor of 0.1 associated with geomagnetic variations in the $\mathrm{Pc} 3$ band (10-45 s period) (Skone, 2008).

In radio astronomy, the Murchison Widefield Array (MWA) in Australia and the LOw Frequency ARray (LOFAR) (e.g., Bastian, 2004) in Europe are new generation telescopes that operate over the $70-300 \mathrm{MHz}$ band and are regarded as technology "demonstrators" for the Square Kilometre Array (SKA) (e.g., Carilli and Rawlings, 2004). These instruments consist of spatial antenna arrays that provide improved resolution using interferometry methods (e.g., Pawsey and Bracewell, 1956). The LOFAR is sensitive to variations in TEC less than a fraction of a percent (Kassim et al., 1993). Horizontal gradients of $0.1 \%$ TEC per km produce 1 radian differential phase over a $10 \mathrm{~km}$ baseline. Using LOFAR to detect ionosphere disturbances and waves has also been discussed by Gaussiran et al. (2004) while TEC data obtained from GPS receivers will assist in the calibration of the MWA. The MWA antenna network has a maximum baseline around $3 \mathrm{~km}$ while the SKA has planned baselines over $3000 \mathrm{~km}$. The performance of these instruments depends on the quality of data obtained during calibration cycles and a large amount of research has focussed on removing effects related to ionosphere disturbances (e.g., Thompson et al., 2001; Erickson et al., 2001).

For large spatial antenna arrays the received signals traverse the ionosphere at different locations and may also have different slant angles. A differential phase (phase difference) arises when the TEC differs along the different signal traversal paths. Radio telescope antenna arrays that depend on interferometry techniques need to calibrate out relative phase differences at each antenna caused by spatial variations in the ionosphere TEC (Hinder and Ryle, 1971). GPS methods use the phase information from the L1 (1575.42 MHZ) and L2 (1227.60 MHz) frequencies and details of GPS based methods for estimating TEC are readily available (e.g., Erickson et al., 2001). In this paper we use recent results from ULF wave research to provide estimates of the changes in TEC due to Pc3-4 activity in the ionosphere to inform GPS applications and we present differential phase estimates expected from ULF wave activity over ULF spatial scale sizes for radio astronomy relevant signals.

\section{ULF waves and the ionosphere}

The near-Earth space environment extends from the ionosphere into the plasmasphere and magnetosphere. Beyond this, the solar magnetic field and solar wind dominate the dynamics. The composition and dynamics of the ionosphere are known to be directly influenced by solar radiation. There are less direct processes that produce electric fields in the ionosphere that also alter the electron density, including ULF waves. The interaction between the solar wind and magne- tosphere provides a rich source for ULF wave energy and the properties of ULF perturbations that propagate through a magnetised plasma were derived by Hannes Alfvén (Alfvén and Fälthammar, 1963). In most of the magnetosphere the plasma is regarded as "cold" ( $10 \mathrm{eV}$ or less) and the two relevant ULF Alfvén waves are the fast or compressional mode and the transverse or shear Alfvén mode. These two wave modes can exhibit quite different spatial structures resulting in a range of differential phases across a spatial antenna array.

Energy from ULF wave activity is always present in the magnetosphere and its inner boundary, the ionosphere. Understanding the generation and energy propagation pathways and processes of ULF waves in the magnetosphere has been an active research field since the 1950s as in-situ measurements became available. Examples of reviews of ULF wave research may be found in Orr (1973), Yumoto (1985), Takahashi (1991), Allan and Poulter (1992), and Takahashi et al. (2006). From these efforts, we know that ULF wave amplitudes vary with the frequency of oscillation, latitude of detection on Earth, wave mode, interplanetary magnetic field properties and geomagnetic activity. Historically, ULF wave properties have been deduced from ground-based magnetometer array time series, supplemented by magnetic perturbation measurements from various spacecraft. While other instruments such as HF radars, Doppler sounders, electric field and particle detectors now provide important ULF wave information, extensive magnetometer arrays are still the major data source for ULF wave research. Limitations and advantages of various ULF wave detection instrumentation is related to the spatial scale size of the perturbation and will be discussed in this paper in due course.

The McIlwain number (McIlwain, 1961), L, is used to identify the latitude in ULF wave research. The $L$ parameter is defined by the distance in Earth radii from the Earth centre to the equatorial crossing location in space of a given geomagnetic field line. For example, the present SKA proposal is to locate the array at low latitudes either in South Africa or Australia in radio quiet areas. The location of the MWA and the proposed centre of the SKA in Australia is $38.6^{\circ}$ south (geomagnetic latitude) with $\mathrm{L}=1.6$. Additional antennae in the SKA may be located at latitudes as high as $\mathrm{L}=2.5$ and $3500 \mathrm{~km}$ away on the east coast of the continent. The centre core for LOFAR in the Netherlands is around $\mathrm{L}=2.4$.

The amplitude and spectral content of ULF waves recorded on the ground are related to the latitude due to properties of the shear Alfvén mode which forms field line resonances (FLRs). Basically, the direction of energy propagation for the shear Alfvén mode is along the geomagnetic field in space where reflection at the north and south ionospheres yield resonant structures analagous to a stretched string (Sugiura and Wilson, 1964; Cummings et al., 1969). The ULF resonant frequencies and associated enhanced amplitudes depend on the geomagnetic field strength, plasma mass loading and geomagnetic field line length. Higher frequencies 
are favoured at lower latitudes. Typical FLR frequencies at $\mathrm{L}=1.8$ are around $50 \mathrm{mHz}$ while FLRs at $\mathrm{L}=2.8$ often contain multiple harmonics with the fundamental around 10 to $15 \mathrm{mHz}$ (Waters et al., 1991a).

Fluctuations in TEC associated with properties of ULF waves have been known for some time (Rishbeth and Garriott, 1964; Davies and Hartman, 1976; Okuzawa and Davies, 1981) and the possible physical mechanisms were discussed by Poole and Sutcliffe (1987). The TEC evaluated along the line between a source $(S)$ and receiver $(R)$ is given by

$\mathrm{TEC}=\int_{S}^{R} N_{e} d z$

while temporal variations in the TEC are described by (Poole and Sutcliffe, 1987)

$\frac{\partial \mathrm{TEC}}{\partial t}=\int_{S}^{R} \frac{\partial N_{e}}{\partial t} d z$

with the electron continuity equation (Rishbeth and Garriott, 1969)

$\frac{\partial N_{e}}{\partial t}=q-l-\boldsymbol{v} \cdot \nabla N_{e}-N_{e} \nabla \cdot \boldsymbol{v}$

where $q$ and $l$ are the electron production and loss rates respectively and $\mathbf{v}$ is the plasma velocity from the ULF perturbation. Assuming $q$ and $l$ are negligible (or equal) then the variations in TEC arise from the remaining advection and divergence terms in Eq. (3).

Variations in TEC along the signal path introduce time/phase delays for high frequency EM wave propagation through the ionosphere. These may be estimated from the resulting perturbations in the refractive index using the usual Appleton-Hartree relations (e.g., Stix, 1962)

$n^{2}=1-\frac{X}{1-i Z-\left(\frac{Y_{T}^{2}}{2(1-X-i Z)}\right) \pm\left(\frac{Y_{T}^{4}}{4(1-X-i Z)^{2}}+Y_{L}^{2}\right)^{1 / 2}}$

for $X=\frac{\omega_{N}^{2}}{\omega^{2}}, Y_{L}=\frac{\omega_{B} \cos \theta}{\omega}, Y_{T}=\frac{\omega_{B} \sin \theta}{\omega}, Z=\frac{\nu}{\omega}, \omega_{N}=\frac{N_{e} e^{2}}{\epsilon_{0} m_{e}}$, $\omega_{B}=\frac{e B_{0}}{m_{e}}$. The refractive index, $n$, depends on $\theta$, the angle between the HF wave propagation direction and the geomagnetic field $\left(B_{0}\right), v$, the electron collision frequency, $\omega_{N}$, the plasma frequency and $\omega_{B}$, the electron gyro-frequency.

The link between TEC variations and ULF waves is through the plasma velocity in Eq. (3). However, measurements of ULF waves are most commonly obtained using ground magnetometer data. We therefore require a way to obtain the ULF wave fields throughout the ionosphere from the ground level ULF magnetic field data. This has recently become possible through the development of ULF wave models that allow for the geomagnetic dip angles and both shear and fast Alfvén mode incident waves at low latitudes (Sciffer and Waters, 2002; Sciffer et al., 2005).

Consider a coordinate system where $X$ is northward, $Y$ is westward and $Z$ is radially outward from the surface of the
Earth. The geomagnetic field, $\mathbf{B}_{\mathbf{0}}$, lies in the $X Z$ plane at an angle, $I$ to the horizontal (see Fig. 1 of Sciffer and Waters, 2002). ULF wave energy is described as an electromagnetic disturbance and the relevant Maxwell equations are

$$
\begin{gathered}
\nabla \times \mathbf{E}=-\frac{\partial \mathbf{B}}{\partial t} \\
\nabla \times \mathbf{H}=\mathbf{J}+\frac{\partial \mathbf{D}}{\partial \mathbf{t}}
\end{gathered}
$$

where the current density, $\mathbf{J}$ and magnetic flux density, $\mathbf{B}$ are given by

$$
\begin{aligned}
\mathbf{J} & =\bar{\sigma} \mathbf{E} \\
\mathbf{B} & =\mu \mathbf{H}
\end{aligned}
$$

For zero background electric field, $\left(\mathbf{E}_{\mathbf{0}}=\mathbf{0}\right)$ the zero and first order perturbation fields are

$$
\begin{aligned}
& \mathbf{B}=\boldsymbol{B}_{0}+\mathbf{b}=\left(\boldsymbol{B}_{\mathbf{0}} \cos (\mathbf{I}), \mathbf{0}, \boldsymbol{B}_{\mathbf{0}} \sin (\mathbf{I})\right)+\left(\boldsymbol{b}_{\mathbf{x}}, \boldsymbol{b}_{\mathbf{y}}, \boldsymbol{b}_{\mathbf{z}}\right) \\
& \mathbf{E}=\mathbf{e}=\left(\mathbf{e}_{\mathbf{x}}, \mathbf{e}_{\mathbf{y}}, \mathbf{e}_{\mathbf{z}}\right)
\end{aligned}
$$

Assuming ULF wave fields with horizontal spatial and time dependence of the form $e^{i\left(k_{x} x+k_{y} y-\omega t\right)}$, the governing equations are

$$
\begin{aligned}
\frac{\partial \boldsymbol{e}_{x}}{\partial z}= & -\frac{i k_{x} \epsilon_{31}}{\epsilon_{33}} \boldsymbol{e}_{x}-\frac{i k_{x} \epsilon_{32}}{\epsilon_{33}} \boldsymbol{e}_{y}+\frac{i k_{x} k_{y} c^{2}}{\epsilon_{33} \omega} b_{x}+i\left(\omega-\frac{k_{x}^{2} c^{2}}{\epsilon_{33} \omega}\right) b_{y} \\
\frac{\partial e_{y}}{\partial z}=- & \frac{i k_{y} \epsilon_{31}}{\epsilon_{33}} e_{x}-\frac{i k_{y} \epsilon_{32}}{\epsilon_{33}} e_{y}-i\left(\omega-\frac{k_{y}^{2} c^{2}}{\epsilon_{33} \omega}\right) b_{x}-\frac{i k_{x} k_{y} c^{2}}{\epsilon_{33} \omega} b_{y} \\
\frac{\partial b_{x}}{\partial z}= & -i\left[\frac{k_{x} k_{y}}{\omega}+\frac{\omega}{c^{2}}\left(\epsilon_{21}-\frac{\epsilon_{23} \epsilon_{31}}{\epsilon_{33}}\right)\right] e_{x} \\
& +i\left[\frac{k_{x}^{2}}{\omega}-\frac{\omega}{c^{2}}\left(\epsilon_{22}-\frac{\epsilon_{23} \epsilon_{32}}{\epsilon_{33}}\right)\right] e_{y}-\frac{i k_{y} \epsilon_{23}}{\epsilon_{33}} b_{x} \\
& +\frac{i k_{x} \epsilon_{23}}{\epsilon_{33}} b_{y} \\
\frac{\partial b_{y}}{\partial z}= & -i\left[\frac{k_{y}^{2}}{\omega}-\frac{\omega}{c^{2}}\left(\epsilon_{11}-\frac{\epsilon_{13} \epsilon_{31}}{\epsilon_{33}}\right)\right] e_{x} \\
& +i\left[\frac{k_{x} k_{y}}{\omega}+\frac{\omega}{c^{2}}\left(\epsilon_{12}-\frac{\epsilon_{13} \epsilon_{32}}{\epsilon_{33}}\right)\right] e_{y}+\frac{i k_{y} \epsilon_{13}}{\epsilon_{33}} b_{x} \\
& -\frac{\epsilon_{33} \epsilon_{13}}{b_{y}}
\end{aligned}
$$

The $\epsilon_{i j}$ are elements of the dielectric tensor, $\bar{\epsilon}$, which is related to the conductivity tensor, $\bar{\sigma}$, by (Zhang and Cole, 1994)

$\bar{\epsilon}=\bar{I}+\frac{i}{\epsilon_{0} \omega} \bar{\sigma}$

The elements of the conductivity tensor are functions of altitude and were computed according to the expressions in 

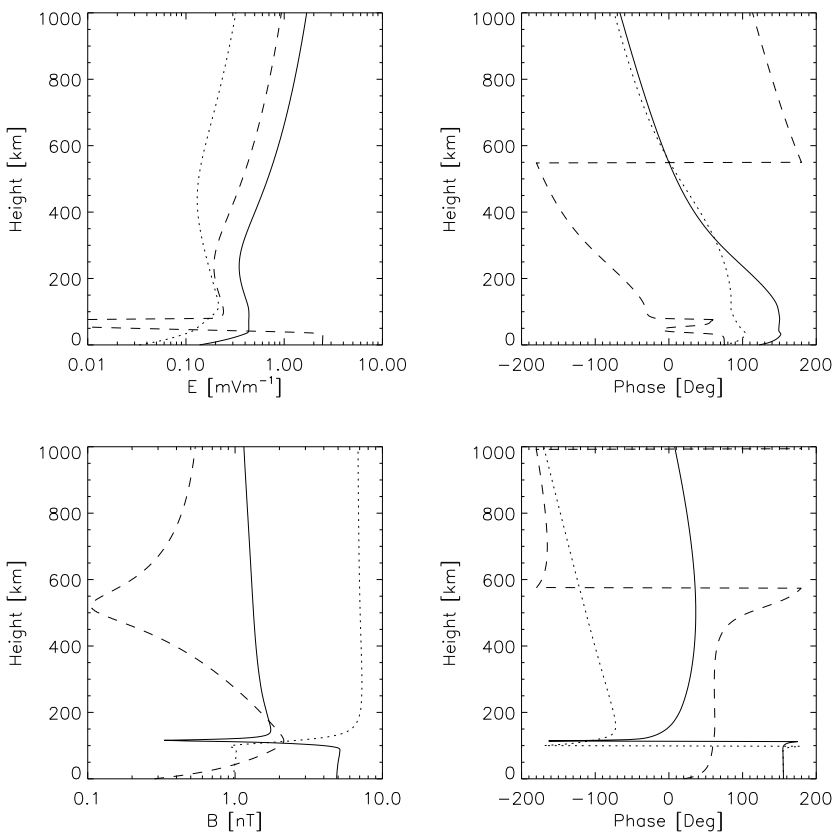

Fig. 1. ULF wave fields at $L=1.6$ for local midday summer. The solid lines are the $\mathrm{X}$ (north-south), the dotted lines are the $\mathrm{Y}$ (eastwest) and the dashed lines are the $\mathrm{Z}$ (vertical) ULF field components. The values for the ULF wave model were $f=50 \mathrm{mHz}$, $k_{x}=3.5 \times 10^{-6} \mathrm{~m}^{-1}, k_{y}=7.0 \times 10^{-7} \mathrm{~m}^{-1}$ with an incident shear Alfvén mode at $1000 \mathrm{~km}$ altitude.

Prince and Bostick (1964). Equations (11) to (14) represent a system of four, first order differential equations. To complete the set, the $e_{z}$ and $b_{z}$ ULF wave components are

$e_{z}=-\frac{\epsilon_{31}}{\epsilon_{33}} e_{x}-\frac{\epsilon_{32}}{\epsilon_{33}} e_{y}+\frac{k_{y} c^{2}}{\epsilon_{33} \omega} b_{x}-\frac{k_{x} c^{2}}{\epsilon_{33} \omega} b_{y}$

$b_{z}=-\frac{k_{y}}{\omega} e_{x}+\frac{k_{x}}{\omega} e_{y}$

and we require four boundary conditions to solve the system. Two of the boundary conditions are specified at the ground. We assume the Earth is a uniform, homogeneous conductor of finite conductivity. The ULF waves decay in amplitude in this medium and are described by

$$
\begin{aligned}
& \frac{\partial e_{x}}{\partial z}-\gamma\left(\sigma_{g}, k_{x}, k_{y}, \omega\right) e_{x}=0 \\
& \frac{\partial e_{y}}{\partial z}-\gamma\left(\sigma_{g}, k_{x}, k_{y}, \omega\right) e_{y}=0
\end{aligned}
$$

where $\gamma$ specifies the ground to be a uniform medium with conductivity, $\sigma_{g}=10^{-2} \mathrm{Mho} / \mathrm{m}$. The top boundary was set at $1000 \mathrm{~km}$ altitude where ideal magnetohydrodynamic (MHD) conditions were assumed. The model allows for the existence of both the shear Alfvén and fast mode waves up to the top boundary. Details of the derivation for this type of boundary condition are given by Sciffer et al. (2005).
Equations (11) to (14) were solved using a second order finite differencing scheme and the Numerical Algorithms Group (NAG) package FO4ADF. The composition of the atmosphere was calculated from the thermosphere model based on satellite mass spectrometer and ground-based incoherent scatter data (MSISE90) (Hedin, 1991). The ionosphere composition was obtained using the International Reference Ionosphere (IRI2007) model and $\mathbf{B}_{\mathbf{0}}$ was obtained from the International Geomagnetic Reference Field (IGRF2005).

\section{Results}

The geographic coordinates for the proposed site of the main hub of the radio astronomy large antenna array in Western Australia are $26.9^{\circ} \mathrm{S}$ and $116.6^{\circ} \mathrm{E}$. Representative profiles of the ULF wave fields vertically through the ionosphere for this location ( $\mathrm{L}=1.6)$ at 12:00 MLT during summer conditions (31 January 2005) are shown in Fig. 1. The ULF wave amplitudes have been scaled to give a $5 \mathrm{nT}$ magnitude calculated from the three components of the perturbation magnetic field at the ground, i.e. $\sqrt{b_{x, g}^{2}+b_{y, g}^{2}+b_{z, g}^{2}}=5 \mathrm{nT}$. The horizontal spatial structure of the ULF wave must be specified in the model. The azimuthal (east-west) scale size may be estimated using the m-number defined by Olson and Rostoker (1978) as

$m=\frac{2 \pi R_{E} \Delta \phi}{360 S} \cos \lambda$

where $R_{E}$ is the Earth radius and $\Delta \phi$ is the measured phase difference between ULF signals detected at two azimuthally spaced locations separated by a distance $S \mathrm{~km}$ at a colatitude of $\lambda$. Measurements of m-numbers for ULF perturbations at low latitudes from ground magnetometer data have yielded values around 3 to 4 (Ostwald et al., 1993). For $\mathrm{L}=1.6, m=4$ translates to an azimuthal wave number, $k_{y}=$ $7.0 \times 10^{-7} \mathrm{~m}^{-1}$. The north-south spatial structure is complicated by resonance effects (FLRs) that enhances the amplitude and reduces the spatial scale size. One approximation is to treat the resonant profile as a gaussian shaped spectrum of wave numbers and select the peak in this k-space.

For the moment, assume that resonance effects confine the north-south structure by a factor of 5 times the azimuthal structure. The effects of the horizontal spatial structure of ULF waves on the ionosphere TEC are discussed later. For these parameters, the ULF magnetic fields in Fig. 1 show the characteristic transition from a $b_{y}$ dominant oscillation in the magnetosphere to a $b_{x}$ component at the ground. This arises from the ability of the shear Alfvén mode to carry field aligned current in the magnetosphere $(\nabla \times \mathbf{b} \neq 0)$ while the neutral atmosphere does not support electric current (Hughes, 1983). These magnetic field oscillations may be related to values measured using ground magnetometers by the appropriate linear scaling. 
The ULF wave fields drive the plasma velocities to give the advection and divergence terms in Eq. (3). The advection term describes variations in $N_{e}$ from movement of existing ionisation gradients (Poole and Sutcliffe, 1987). The $\nabla N_{e}$ term has horizontal and vertical gradient components. If there are no horizontal gradients then the advection term becomes $v_{z} \frac{\partial N_{e}}{\partial z}$. If $N_{e}$ is zero at both the HF signal receive (ground) and source locations then the integral of $\frac{\partial N_{e}}{\partial z}$ over the receive/source path is zero. This is why only the divergence term was used by Pilipenko and Fedorov (1995) to estimate changes in TEC from ULF wave activity.

For GPS applications, $N_{e}$ is not zero at geostationary heights but it is much smaller than the F layer peak so the advection integral is much smaller than the integrated divergence term. However, there are often horizontal gradients in $N_{e}$ and these contribute to the advection term. Large scale horizontal gradients in $N_{e}$ occur around sunrise and sunset, at high latitudes due to auroral processes and are associated with the equatorial anomaly. Smaller scale horizontal gradients are known to be produced by other ionosphere disturbances such as TIDs and gravity waves. As pointed out by Poole and Sutcliffe (1987), horizontal gradient contributions from the advection term are comparable with the divergence term for Pc3-4 ULF induced TEC variations of $0.1 \%$ change in TEC per km, estimated using a ULF horizontal electric field of $1 \mathrm{mVm}^{-1}$ in the direction of the maximum gradient.

For a $70 \mathrm{MHz}$ signal propagating vertically through the ionosphere, the associated differential phase horizontally across the ULF perturbation structure for the parameters in Fig. 1, from the divergence term only is $8^{\circ}$. However, this value may be much larger depending on the parameters of the ULF disturbance and properties of $N_{e}$. There are a number of assumptions that have been used in the literature for estimating ULF wave fields from the magnetosphere to the ground. Many assume vertical geomagnetic fields, electrostatic ionosphere reflection physics and only shear Alfvén mode incident waves into the system. ULF waves interact with the anisotropic ionosphere plasma and produce fast mode oscillations, the source of plasma compression and the main process identified by Poole and Sutcliffe (1987) in the divergence term of Eq. (3). A full analysis of the parameters that determine ULF mode conversion has recently been discussed by Sciffer et al. (2005). The geomagnetic dip angle, wave frequency, spatial scale and ULF wave mode mix all contribute to the final wave structure and this is the first application of this improved ULF wave model to the estimation of associated TEC perturbations.

The first parameter we consider is the ULF wave mode mix. Figure 1 shows the ULF fields for an incident shear Alfvén mode only, a common assumption in the literature that is argued on the basis of choosing the FLR frequency. However, FLRs are regularly observed over all latitudes greater than $L \approx 1.3$ and persist throughout the whole daytime and therefore require energy input to sustain oscillation.
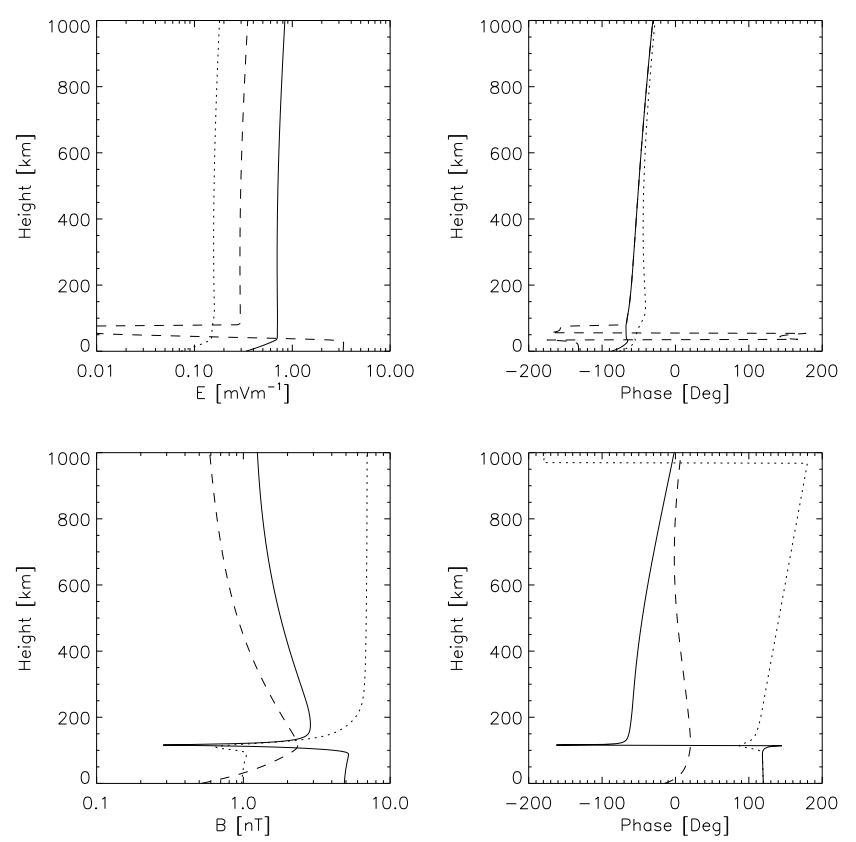

Fig. 2. ULF wave fields at $\mathrm{L}=2.4$ for local midday winter (North Hemisphere). The solid lines are the $X$ (north-south), the dotted lines are the Y (east-west) and the dashed lines are the $\mathrm{Z}$ (vertical) ULF field components. The parameters for the ULF wave model were $f=15 \mathrm{mHz}, k_{x}=3.5 \times 10^{-6} \mathrm{~m}^{-1}, k_{y}=7.0 \times 10^{-7} \mathrm{~m}^{-1}$ with an incident shear Alfvén mode at $1000 \mathrm{~km}$ altitude.

This energy comes from coupling with fast mode waves and so we expect some mix of the two ULF modes, even at the FLR frequency. Results from the ULF model for the same parameters as Fig. 1 but with the ULF input wave mode mix at $80 \%$ shear Alfvén and $20 \%$ fast mode increases the associated differential phase to $20^{\circ}$ and $0.016 \%$ change in TEC.

Moving now to the Northern Hemisphere, the LOFAR centre is located in the Netherlands near $53^{\circ}$ geographic latitude ( $\mathrm{L}=2.4)$. This higher latitude supports lower ULF first harmonic (fundamental) FLRs around $15 \mathrm{mHz}$. The ULF wave fields for a $100 \%$ incident shear Alfven, $15 \mathrm{mHz}$ oscillation are shown in Fig. 2 for the same date and horizontal spatial structure as Fig. 1 at local noon. For these parameters and a vertically propagating HF signal, the differential phase from the divergence term is $11^{\circ}$ with a $0.01 \%$ change in TEC.

Figures 1 and 2 show the ULF fields for local noon where, for the $5 \mathrm{nT}$ magnetic field magnitude at the ground, the electric fields are around $1 \mathrm{mVm}^{-1}$. The ratio of the ULF electric and magnetic fields in the magnetosphere depends on the Alfvén speed. This suggests that the ULF fields will depend on diurnal changes in $N_{e}$ which may be illustrated by considering the ULF fields and associated differential phase and fractional TEC around sunrise. Using the same ULF parameters as Fig. 2 and running the IRI model for 06:00 LT gives the ULF fields shown in Fig. 3. For this case, the differential 

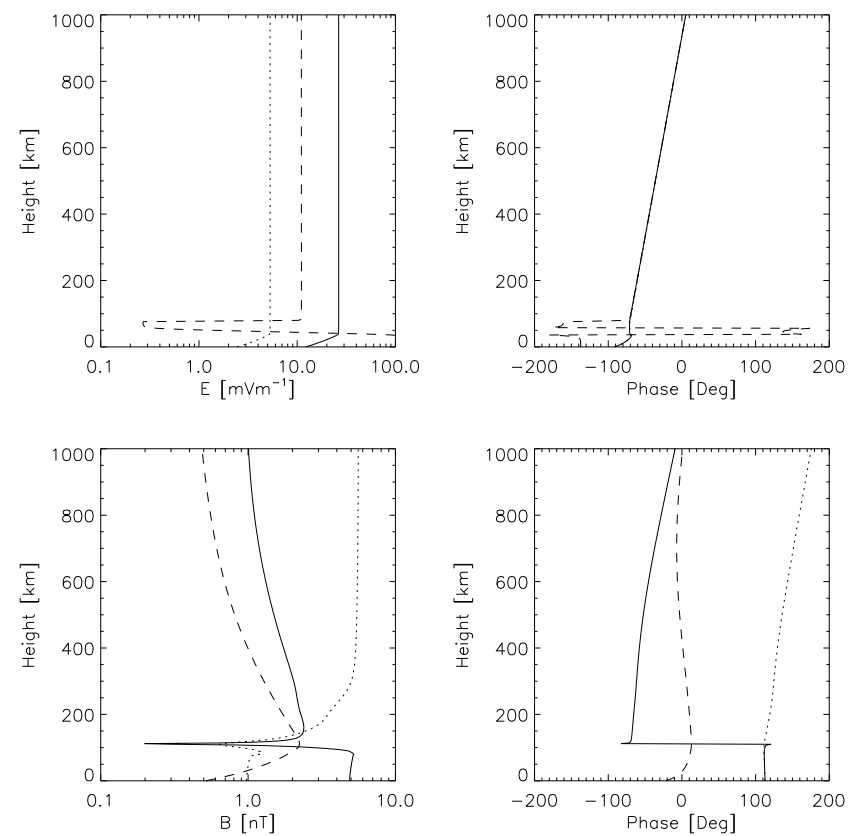

Fig. 3. ULF wave fields at $L=2.4$ for early morning (06:00 LT) winter (North Hemisphere). The solid lines are the $X$ (north-south), the dotted lines are the $\mathrm{Y}$ (east-west) and the dashed lines are the $\mathrm{Z}$ (vertical) ULF field components. The values for the ULF wave model were $f=15 \mathrm{mHz}, k_{x}=3.5 \times 10^{-6} \mathrm{~m}^{-1}, k_{y}=7.0 \times 10^{-7} \mathrm{~m}^{-1}$ with an incident shear Alfvén mode at $1000 \mathrm{~km}$ altitude.

phase across the horizontal ULF structure due to the divergence term is $227^{\circ}$. Most of this arises from the presence of a large vertical gradient in $v_{z}$ in the $\mathrm{E}$ region.

In addition to the divergence term, the early morning (and sunset) case must also consider horizontal gradients from the advection term. While Poole and Sutcliffe (1987) acknowledged that the advection term might be important, depending on the gradients and the ULF electric field magnitude, previous estimates have discarded contributions from this term. For the same parameters as Fig. 3, the IRI model was used to estimate the east-west gradients in $N_{e}$ as a function of altitude. These data were multiplied by the ULF $v_{y}$ and integrated to yield the advection term. For the parameters in Fig. 3 the divergence term is 4 times greater than the advection term and the differential phase for the $70 \mathrm{MHz}$ signal increases to $283^{\circ}$ with a $2.2 \%$ change in TEC. However, it is not yet clear in ULF wave research that a $100 \%$ incident shear Alfvén wave mode is realistic. For $90 \%$ input shear Alfvén mode mix the differential phase from both the divergence and advection contributions is $105^{\circ}$ with a $0.8 \%$ change in TEC while for an $80 \%$ shear Alfvén ULF mode mix, the differential phase and change in TEC are $54^{\circ}$ and $0.5 \%$, respectively.

\section{Discussion}

The magnitude of the change in ionosphere TEC due to ULF wave activity depends on properties of the ULF disturbance. While many of the ULF wave properties can be directly measured there are a number that require further research in order to quantify them more accurately. The amplitudes of the magnetic field perturbations at the ground over the ULF band are easily measured. A number of studies of low latitude ULF waves have shown the tendency for the amplitude to increase during the local day time (Waters, 1992; Bloom and Singer, 1995), particularly around noon with a general decrease in amplitude with increasing frequency (Campbell, 1966), except where the local field line resonance enhances the amplitude. Enhanced geomagnetic activity, parameterised by the $K_{p}$ index, increases the amplitude and widens the frequency band of ULF oscillations.

The spatial structure of ULF variations produces a similar spatial variation in $N_{e}$ causing differential phase for interferometry based radio astronomy techniques. While some measurements of ULF wave spatial structures are available, there are details that are not fully understood. The azimuthal structure may be estimated using Eq. (20). Reported m-numbers at low latitudes range from zero up to 15 while most reports seem to favour $m=3$ to 5 (Ostwald et al., 1993; Waters et al., 1991b; Ziesolleck et al., 1993). These measurements come from ground based magnetometer arrays and assume a dominant value for the spatial structure, $k$. The ULF azimuthal spatial structure should be more realistically described by a $k$ spectrum.

There is an upper limit to the m-number of ULF signals that can be detected using ground magnetometers. This is related to the exponential decay of the ULF signal as it converts into an EM wave in the atmosphere. The ULF signals are exponentially attenuated for spatial scale sizes of the order of the ground to ionosphere E-region height (Hughes and Southwood, 1976; Ponomarenko et al., 2001) so that the ionosphere/atmosphere acts as a spatial, low pass filter. This means that it is possible that small scale ULF perturbations may be present in the ionosphere but little ULF signal will be detected at the ground. At present, ground magnetometer measurements of ULF waves are used to ensure that variations seen in the ionosphere data are due to ULF wave activity rather than by other processes such as gravity waves or TIDs (Menk et al., 2007). This is clearly not possible for the small spatial scale ULF waves. Measurements of ULF properties in the ionosphere at low latitudes are required in order to determine the properties of small scale ULF activity. Gaussiran et al. (2004) discuss one possible method using LOFAR for obtaining suitable data.

Quantifying the spatial structure in the geomagnetic northsouth direction is more problematic. Around the local field line resonant frequencies, the spatial structure may be measured using the ULF magnetic field amplitude data from an array of north-south spaced magnetometers with the 


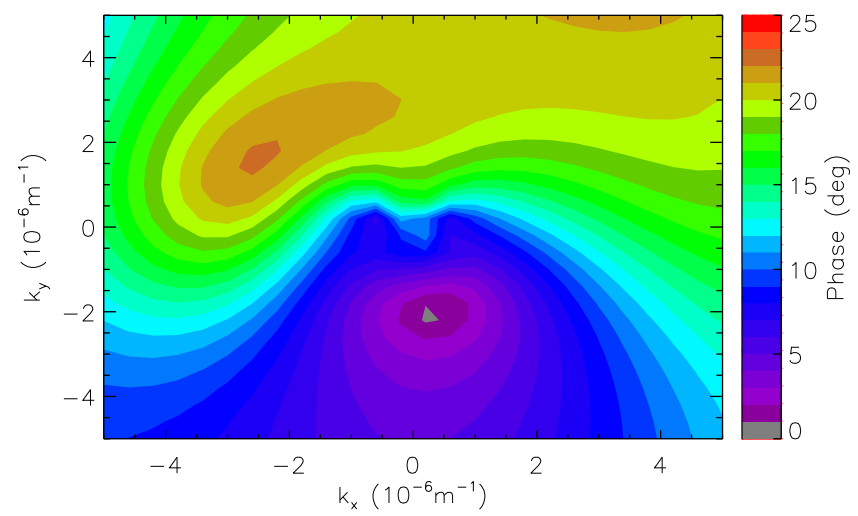

Fig. 4. The differential phases for noon and summer at $\mathrm{L}=1.6$ (South Hemisphere) for a $70 \mathrm{MHz}$ signal due to changes in TEC from a $50 \mathrm{mHz}$ ULF wave as a function of the ULF wave spatial scale size. The ionosphere and neutral atmosphere parameters are the same as those used for Fig. 1. The upper boundary wave mode mix is $80 \%$ shear Alfvén mode.

amplitude versus latitude data approximated by a gaussian function. Fourier transforming this profile yields the spectrum for $k_{x}$. These properties suggest limits on the likely values for $k_{x}$ and $k_{y}$ so we have calculated the ULF wave solutions for a range of ULF spatial structures as shown in Figs. 4 and 5, keeping in mind that the values for $k_{x}$ derived from ground based magnetometer data are also influenced by the ionosphere/atmosphere spatial low pass filter described above.

One final parameter is required to yield a ULF wave solution. This is the ULF wave mode mix at the upper boundary of the model. It is generally accepted that for the larger spatial scale waves seen at the ground, the fast mode provides the energy source for the excitation of FLRs. Around the local FLR frequencies we would expect the shear Alfvén mode to dominate the mode mix. At low latitudes, a particular geomagnetic field trace has a greater proportion of the path affected by ionosphere plasma compared with a field trace from higher latitudes. This tends to dampen FLRs at the lower latitudes, increasing the resonance width (spatial scale size) which reduces the differential phase over a given distance. Efforts to obtain more realistic estimates of the ULF wave mode mix are presently being pursued using higher dimensional ULF wave modeling studies (e.g., Waters and Sciffer, 2008).

In order to determine how the ULF spatial structure affects changes in $N_{e}$, the differential phase and percentage change in TEC were calculated over a range of $k_{x}, k_{y}$ values and ULF mode mixtures. For a $70 \mathrm{MHz}$ EM signal passing vertically through the ionosphere, the differential phase over distances of half the ULF horizontal wavelengths introduced by a $50 \mathrm{mHz}$ perturbation with $80 \%$ shear Alfvén mode mix at the upper boundary $(1000 \mathrm{~km})$ is shown in Fig. 4. There is a hint of symmetry with $k$. In fact, the plot would be sym-

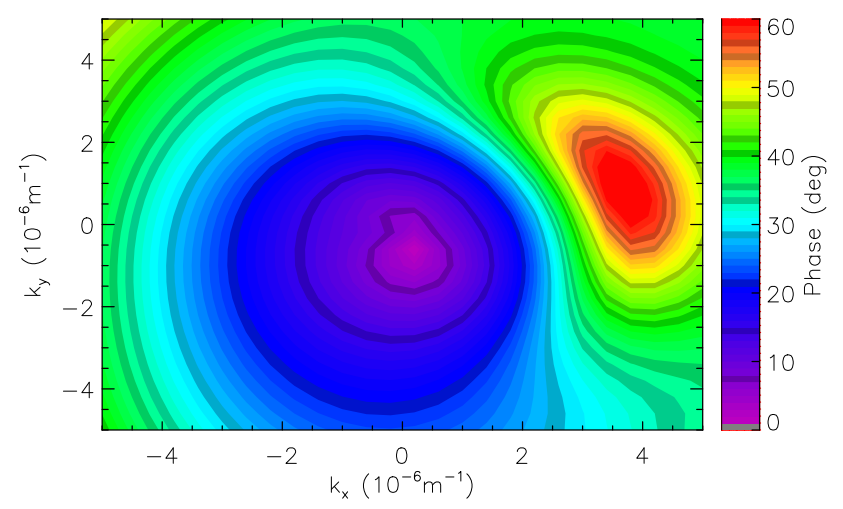

(a)

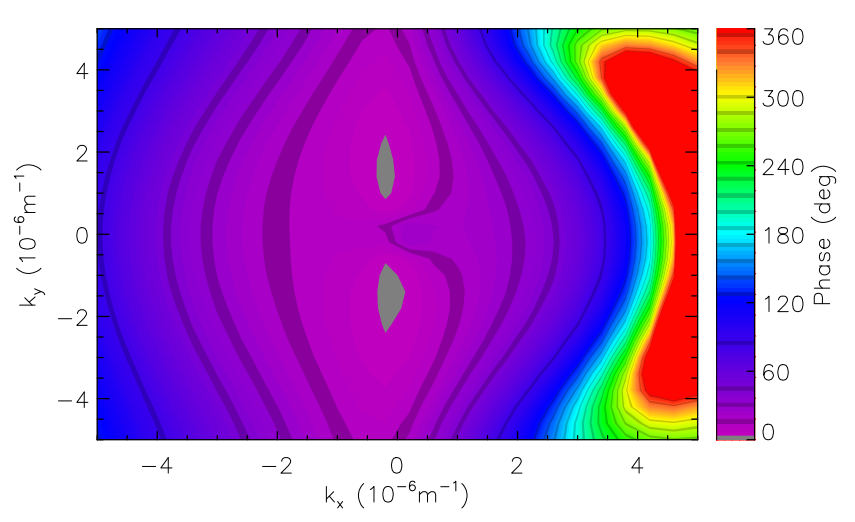

(b)

Fig. 5. The variation in differential phase for a $70 \mathrm{MHz}$ signal due to changes in TEC from a $15 \mathrm{mHz}$ ULF wave with ULF wave mix of $80 \%$ shear Alfvén mode at $1000 \mathrm{~km}$ altitude, as a function of the ULF wave spatial scale size. (a) Local noon using the divergence term only, (b) local morning (06:00 LT) including the advection and divergence terms.

metric if the geomagnetic field dip angle was vertical. Asymmetries in the response of the ionosphere to ULF wave fields versus spatial scale arise from the relationship between the horizontal ULF wave number and the horizontal projection of the geomagnetic field (north-south direction), as discussed by Sciffer et al. (2005). The differential phase is up to $22^{\circ}$ but this excludes the advection term, ignoring horizontal gradients in $N_{e}$. For GPS applications, the percentage change in TEC can also be obtained from Fig. 4 by changing the phase axis to \% TEC and setting the maximum value at $0.02 \%$.

The ULF wave amplitudes generally decrease with increasing frequency (Campbell, 1966) and typical L=1.6 amplitudes are a few nT. An additional amplitude effect at the higher ULF frequencies, such as $50 \mathrm{mHz}$, is the inductive response of the ionosphere discussed by Yoshikawa et al. (2002) and Sciffer et al. (2005) which may reduce the ULF amplitudes sensed at the ground. Studies of the role of this inductive effect and under what conditions it becomes important are part of present ULF wave research. 
For the lower ULF frequency $(15 \mathrm{mHz})$, amplitudes at the ground easily reach $5 \mathrm{nT}$. Figure 5 shows the differential phase for the $15 \mathrm{mHz}, \mathrm{L}=2.4$, Northern Hemisphere case. Figure $5 \mathrm{a}$ is for local noon with no advection term contribution. The maximum differential phase and associated maximum percentage TEC is $62^{\circ}$ and $0.07 \%$, respectively. Figure $5 \mathrm{~b}$ shows the differential phase for 06:00 local time at $\mathrm{L}=2.4$, including the advection contribution. The TEC for dawn compared with noon reduces by a factor of 10 , increasing the ULF electric fields. The maximum differential phase is found for large positive values for $k_{x}$ and involves almost 5 cycles $\left(1770^{\circ}\right)$.

Large variations in TEC may been observed for very small to zero ULF magnetic field amplitudes seen by ground magnetometers due to the spatial filtering. Ground magnetometer data provide many insights into the properties of ULF waves. However, to obtain more complete information, ULF data are required over a spatial region in the ionosphere. A few spatial measurements of ULF waves in the ionosphere have been obtained using Dopppler sounding techniques (Yeoman et al., 2000; Baddeley et al., 2005; Waters et al., 2007). ULF scale sizes down to $50 \mathrm{~km}$ have been observed for latitudes near $\mathrm{L}=6$ (Baddeley et al., 2005). These smaller scale ULF events are thought to be generated through wave-particle interactions associated with magnetospheric ring current ions. If this is the case, the occurrence of these small scale waves may be limited in latitude.

Doppler sounder measurements of ULF waves at low latitudes (L 1.8-2.8) have revealed resonance structures with scale sizes smaller than those seen in ground magnetometer data (Menk et al., 2007). These observations and recent modeling show that ULF scale sizes in the ionosphere at low latitudes may be routinely as small as $150 \mathrm{~km}$, half those determined from ground based data (Waters and Sciffer, 2008). The spatial scale size appears to be a function of the ionosphere Pedersen conductance.

Another recent technique that is being used to understand the relationship between ULF wave properties in the ionosphere compared with those seen at the ground involves overthe-horizon radars (OTHR). The Super Dual Auroral Radar Network (SuperDARN) is an international consortium that operate and conduct research on the data returned by 21 radars that ring the northern and southern auroral regions. Technical details of the radars are described in Greenwald et al. (1985). The Doppler shifts in the backscattered radar returns provide estimates of the plasma velocity. Since the received radar signals refract and do not pass through the ionosphere into space, this method provides information on both the advection and divergence mechanisms.

Signatures of ULF waves have been seen in both the ionosphere and ground scatter returns (Walker et al., 1979; Fenrich et al., 1995; Yeoman et al., 2000; Ponomarenko et al., 2001; Baddeley et al., 2002; Ponomarenko et al., 2005; Fenrich et al., 2006; Waters et al., 2007). The radars can detect ULF signatures when the spatial scale prevents the signal appearing at the ground (Yeoman et al., 2000; Yeoman and Wright, 2001; Baddeley et al., 2002). Furthermore, data from the radars are available at much smaller spatial resolution $(\sim 45 \mathrm{~km})$ and over large regions of water where ground magnetometers do not exist. As more radars are constructed with overlapping fields of view and at lower latitudes more precise information will become available on the spatial structure of ULF waves, allowing more detailed knowledge of the relationship between the ground and ionosphere signatures of ULF disturbances in the geomagnetic field.

\section{Conclusion}

The variations in the phase of HF signals passing through the ionosphere depends on the temporal variations in TEC along the propagation path. We have shown that ULF waves, incident from the magnetosphere and through their interaction with the ionosphere, may cause differential phase variations in $70 \mathrm{MHz}$ signals from a few degrees up to 5 cycles over the scale size of the ULF disturbance.

The interaction of ULF waves with the ionosphere plasma is a complex process that involves the ULF frequency, spatial scale, geomagnetic field dip angle, ionosphere conductances and ULF wave mode mix. For small spatial scale ULF activity it is possible that large TEC variations may be observed while the ground magnetometer signal is very small or even absent. The spatial scale size of the ULF disturbance and associated ionosphere/atmosphere screening is responsible for this effect. Further research is required to obtain estimates of the ULF spatial scales and the ULF wave mode mix, particularly at low latitudes. These require in-situ observations within the ionosphere such as those provided by the SuperDARN data.

Acknowledgements. Topical Editor M. Pinnock thanks two anonymous referees for their help in evaluating this paper.

\section{References}

Alfvén, H. and Fälthammar: Cosmical electrodynamics, Oxford University Press, 1963.

Allan, W. and Poulter, E. M.: ULF waves - Their relationship to the structure of the Earth's magnetosphere, Rep. Prog. Phys., 55, 533-598, 1992.

Baddeley, L. J., Yeoman, T. K., Wright, D. M., Davies, J. A., Trattner, K. J., and Roeder, J. L.: Morning sector drift-bounce resonance driven ULF waves observed in artificially-induced HF radar backscatter, Ann. Geophys., 20, 1487-1498, 2002, http://www.ann-geophys.net/20/1487/2002/.

Baddeley, L. J., Yeoman, T. K., and Wright, D. M.: HF doppler sounder measurements of the ionospheric signatures of small scale ULF waves, Ann. Geophys., 23, 1807-1820, 2005, http://www.ann-geophys.net/23/1807/2005/.

Bastian, T. S.: Low-frequency solar radiophysics with LOFAR and FASR, Planet. Space Sci., 52, 1381-1389, 2004. 
Bloom, R. M. and Singer, H. J.: Diurnal trends in geomagnetic noise power in the Pc 2 through Pc 5 bands at low geomagnetic latitudes, J. Geophys. Res., 100, 14943-14953, 1995.

Campbell, W. H.: A review of the equatorial studies of rapid fluctuations in the Earth's magnetic field, Ann. Geophys., 22, 492-500, 1966.

Carilli, C. and Rawlings, S.: Science with the square kilometre array, Elsevier, Amsterdam, Netherlands, 2004.

Cummings, W. D., O'Sullivan, R. J., and Coleman, P. J.: Standing Alfven waves in the magnetosphere, J. Geophys. Res., 74, 778793, 1969.

Davies, K. and Hartman, G. K.: Short-period fluctuations in total columnar electron content, J. Geophys. Res., 81, 3431-3434, 1976.

Erickson, W. C., Perley, R. A., Flatters, C., and Kassim, N. E.: Ionospheric corrections for VLA observations using local GPS data, Astron. Astrophys., 366, 1071-1080, 2001.

Fenrich, F. R., Samson, J. C., Sofko, G., and Greenwald, R. A.: ULF high- and low-m field line resonances observed with the Super Dual Auroral Radar Network, J. Geophys. Res., 100, 21535 21547, 1995.

Fenrich, F. R., Waters, C. L., Connors, M., and Bredeson, C.: Ionospheric signatures of ULF waves: Passive radar techniques, in: Magnetospheric Current Systems, edited by: Takahashi, K., Chi, P., Denton, R. E., and Lysak, R. L., Geophysical Monograph 169, pp. 259-272, American Geophysical Union, Washingtion, D.C., 2006.

Gaussiran, T. L., Bust, G. S., and Garner, T. W.: LOFAR as an ionospheric probe, Planet. Space Sci., 52, 1375-1380, doi:10. 1016.j.pss.2004.09.007, 2004.

Greenwald, R. A., Baker, K. B., Hutchins, R. A., and Hanuise, C.: An HF phased-array radar for studying small-scale structure in the high-latitude ionosphere, Radio Sci., 20, 63-79, 1985.

Hedin, A. E.: Extension of the MSIS thermosphere model into the middle and lower atmosphere, J. Geophys. Res., 96, 1159-1172, 1991.

Hinder, R. and Ryle, M.: Atmospheric limitations to the angular resolution of aperture synthesis radio telescopes, Mon. Not. R. astr. Soc., 154, 229-253, 1971.

Hughes, W. J.: Hydromagnetic waves in the magnetosphere, in: Solar-Terrestrial Physics, edited by: Carovillano, R. L. and Forbes, J. M., D. Reidel Pub. Co., 1983.

Hughes, W. J. and Southwood, D.: The screening of micropulsation signals by the atmosphere and ionosphere, J. Geophys. Res., 81, 3234-3240, 1976.

Kassim, N., Perley, R., Erickson, W., and Dwarakanath, K.: Subarcminute resolution imaging of radio sources at $74 \mathrm{MHz}$ with the very large array, Astron. J., 106, 2218-2228, 1993.

Kelley, M. C.: The Earth's Ionosphere, Plasma Physics and Electrodynamics, Academic Press, Inc., San Diego, California, 1989.

McIlwain, C. E.: Coordinates for mapping the distribution of magnetically trapped particles, J. Geophys. Res., 66, 3681-3691, 1961.

Menk, F. W., Waters, C. L., and Dunlop, S. I.: ULF Doppler oscillations in the low latitude ionosphere, Geophys. Res. Lett., 34, L10104, doi:10.1029/2007GL029300, 2007.

Okuzawa, T. and Davies, K.: Pulsations in the total columnar electron content, J. Geophys. Res., 86, 1355-1363, 1981.

Olson, J. V. and Rostoker, G.: Longitudional phase variation of Pc
4-5 micropulsations., J. Geophys. Res., 83, 2481-2488, 1978.

Orr, D.: Magnetic pulsations within the magnetosphere: A review, J. Atmos. Terr. Phys., 35, 1-50, 1973.

Ostwald, P. M., Menk, F. W., Fraser, B. J., Waters, C. L., and McNabb, P. W.: Spatial and temporal characteristics of 15$100 \mathrm{mHz}$ ULF waves recorded across a low-latitude azimuthal array, Ann. Geophys., 11, 742-752, 1993.

Pawsey, J. L. and Bracewell, R. N.: Radio Astronomy, Q. J. Roy. Meteorol. Soc., 82, 120-361, 1956.

Pilipenko, V. A. and Fedorov, E. N.: Modulation of the total electron content in the ionosphere by geomagnetic pulsations, Geoamgn. Aeron., 34, 516-519, 1995.

Ponomarenko, P. V., Waters, C. L., Sciffer, M. D., and Fraser, B. J.: Spatial structure of ULF waves: Comparison of magnetometer and Super Dual Auroral Radar Network data, J. Geophys. Res., 106, 10509-10517, 2001.

Ponomarenko, P. V., Menk, F. W., Waters, C. L., and Sciffer, M. D.: Pc3-4 ULF waves observed by the SuperDARN TIGER radar, Ann. Geophys., 23, 1271-1280, 2005, http://www.ann-geophys.net/23/1271/2005/.

Poole, A. W. V. and Sutcliffe, P. R.: Mechanisms for observed total electron content pulsations at mid latitudes, J. Atmos. Terr. Phys., 49, 231-236, 1987.

Prince, C. E. and Bostick, F. X.: Ionospheric transmission of transversely propagated plane waves at micropulsation frequencies and theoretical power spectrums., J. Geophys. Res., 69, 32133234, 1964.

Rishbeth, H. and Garriott, O.: Introduction to ionospherice physics, Academic Press, New York, 1969.

Rishbeth, H. and Garriott, O. K.: Relationship between simultaneous geomagnetic and ionospheric oscillations, Radio Sci., 68D, 339-343, 1964.

Schunk, R. W. and Nagy, A. F.: Ionospheres of the terrestrial planets, Rev. Geophys. Space Phys., 18, 813-852, 1980.

Sciffer, M. D. and Waters, C. L.: Propagation of ULF waves through the ionosphere: Analytic solutions for oblique magnetic fields, J. Geophys. Res., 107, 1297-1311, 2002.

Sciffer, M. D., Waters, C. L., and Menk, F. W.: A numerical model to investigate the polarisation azimuth of ULF waves through an ionosphere with oblique magnetic fields, Ann. Geophys., 23, 3457-3471, 2005, http://www.ann-geophys.net/23/3457/2005/.

Skone, C.: Mitigating geomagnetic noise in airborne magnetic surveys using GPS, in: ION GNSS 2008, Savannah, Georgia, USA, 2008.

Stix, T. H.: The theory of plasma waves., McGraw-Hill, New York, 1962.

Sugiura, M. and Wilson, C. R.: Oscillation of the geomagnetic field and associated magnetic perturbations at conjugate points, J. Geophys. Res., 69, 1211-1216, 1964.

Takahashi, K.: ULF waves in the magnetosphere, Rev. Geophys. Suppl., p. 1066, 1991.

Takahashi, K., Chi, P. J., Denton, R. E., and Lysak, R. L. (Eds.): Magnetospheric ULF waves: Synthesis and new directions, Geophysical Monograph 169, AGU, Washington, D.C., 2006.

Thompson, A. R., Moran, J. M., and Swenson, G. W.: Interferometry and synthesis in radio astronomy, Wiley-Interscience, New York, 2001.

Walker, A. D. M., Greenwald, R. A., Stuart, W. F., and Green, C. A.: STARE Auroral Radar Observations of Pc 5 Geomagnetic pulsa- 
tions, J. Geophys. Res., 84, 3371-3388, 1979.

Waters, C. L.: Low latitude geomagnetic field line resonance, $\mathrm{PhD}$ dissertation, University of Newcastle, School of Mathematical and Physical Sciences, 1992.

Waters, C. L. and Sciffer, M. D.: Field line resonant frequencies and ionospheric conductance: Results from a 2-D MHD model, J. Geophys. Res., 113, A05219, doi:10.1029/2007JA012822, 2008.

Waters, C. L., Menk, F. W., and Fraser, B. J.: The resonant structure of low latitude Pc 3 geomagnetic pulsations, Geophys. Res. Lett., 18, 2293-2296, 1991a.

Waters, C. L., Menk, F. W., Fraser, B. J., and Ostwald, P. M.: Phase structure of low latitude Pc3-4 pulsations, Planet. Space Sci., 39, 569-582, 1991b.

Waters, C. L., Yeoman, T. K., Sciffer, M. D., Ponomarenko, P., and Wright, D. M.: Modulation of radio frequency signals by ULF waves, Ann. Geophys., 25, 1113-1124, 2007, http://www.ann-geophys.net/25/1113/2007/.

Yeoman, T. K. and Wright, D. M.: ULF waves with drift resonance and drift-bounce resonance energy sources as observed in artificially-induced HF radar backscatter, Ann. Geophys., 19, 159-170, 2001, http://www.ann-geophys.net/19/159/2001/.
Yeoman, T. K., Wright, D. M., and Chapman, P. J.: High latitude observations of ULF waves with large azimuthal wavenumbers, J. Geophys. Res., 105, 5453-5462, 2000.

Yoshikawa, A., Obana, Y., Shinohara, M., Itonaga, M., and Yumoto, K.: Hall-induced inductive shielding effect on geomagnetic pulsations, Geophys. Res. Lett., 29, 8, doi:10.1029/2001GL013610, 2002.

Yumoto, K.: Low-Frequency Upstream Wave as a Probable Source of Low-Latitude Pc3-4 Magnetic Pulsations, Planet. Space Sci., 33, 239-249, 1985.

Zhang, D. Y. and Cole, K. D.: Some aspects of ULF electromagnetic wave relations in a stratified ionosphere by the method of boundary value problem, J. Atmos. Terr. Phys., 56, 681-690, 1994.

Ziesolleck, C. W. S., Fraser, B. J., Menk, F. W., and McNabb, P. W.: Spatial characteristics of low-latitude Pc3-4 geomagnetic pulsations, J. Geophys. Res., 98, 197-207, 1993. 